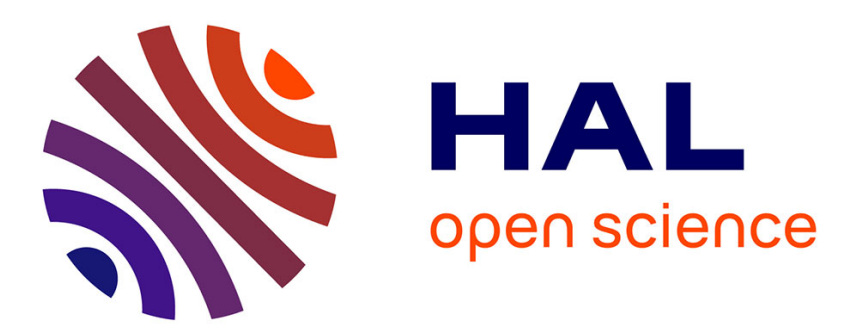

\title{
A wide range constitutive equation for medium and high strength steel
}

B. Goldthorpe, A. Butler, P. Church

\section{To cite this version:}

B. Goldthorpe, A. Butler, P. Church. A wide range constitutive equation for medium and high strength steel. Journal de Physique IV Proceedings, 1994, 04 (C8), pp.C8-471-C8-476. 10.1051/jp4:1994873 . jpa-00253434

\section{HAL Id: jpa-00253434 https://hal.science/jpa-00253434}

Submitted on 1 Jan 1994

HAL is a multi-disciplinary open access archive for the deposit and dissemination of scientific research documents, whether they are published or not. The documents may come from teaching and research institutions in France or abroad, or from public or private research centers.
L'archive ouverte pluridisciplinaire HAL, est destinée au dépôt et à la diffusion de documents scientifiques de niveau recherche, publiés ou non, émanant des établissements d'enseignement et de recherche français ou étrangers, des laboratoires publics ou privés. 


\title{
A wide range constitutive equation for medium and high strength steel
}

\author{
B.D. Goldthorpe, A.L. Butler and P. Church
}

DRA Fort Halstead, Sevenoaks, Kent, U.K.

\begin{abstract}
A Constitutive Equation has been determined for a medium strength steel and has been shown to have identical form to the modified Armstrong Zerilli equation used for iron. The strengthening mechanism in steel appears to act through a constant which is subject to thermal softening through the shear modulus but the part of the model describing thermally activated processes remains almost identical to that observed in iron. These observations suggest the possibility of using this equation as a generic form for a wide class of steels in which the determination of coefficients is simplified considerably.
\end{abstract}

\section{INTRODUCTION}

In recent years there has been a considerable increase in interest in constitutive equations particularly in those with a wide range of application in strain rate, temperature and strain and with a sound physical basis. There are two important reasons for this, firstly because it is now generally appreciated that predictive hydrocode modelling is impossible with inadequate input data and secondly because improvements in analytic theory have shown the need for accurate constitutive relationships.

Constitutive equations developed from basic physical principles have been published by Klepaczco (1) and Follansbee (2) for application to both FCC and BCC metals and Armstrong and Zerilli (3) have published separate BCC and FCC models. The BCC model developed by Armstrong and Zerilli has been modified by Goldthorpe (4) and applied to annealed and explosively shocked iron. This constitutive equation has been shown by Cullis and Church (5) to have very good accuracy over a wide range of all the main variables in the model. The main purpose of the present paper has been to carry out a full determination of a constitutive equation for a medium strength steel and determine whether the model for iron (4) can be used as a basis for a generic form and thus simplify model determination for other steels.

\section{MATERIALS AND EXPERIMENTAL METHODS}

The steel used in this investigation was a quenched and tempered carbon-chromium molybdenum steel with a quasi static yield strength of $800 \mathrm{MPa}$ and was supplied in the form of plate.

The test technique used for the determination of the constitutive equation were tensile tests over a wide range of temperatures and Hopkinson bar tests in compression. 


\section{DETERMINATION OF THE FORM OF THE MODEL}

One of the main problems in the determination of wide range constitutive equations is that in any test that is not perfectly isothermal strain hardening and thermal softening take place simultaneously. Thus it is essential to separate experimentally the main variables of temperature, strain rate and strain if an accurate model is to be determined. A successful method of achieving this was developed by Goldthorpe (4) in which isothermal stress strain curves could be determined under conditions where deformation could range from isothermal to adiabatic. This involved the use of interrupted tests in which a strain increment was applied and the test stopped and the specimen allowed to cool down. If stress measurements are only taken on the early part of this strain increment the temperature rise can be restricted to any desired level but usually less than $5^{\circ} \mathrm{K}$. This procedure can be repeated many times until the desired strain level is reached and a composite curve built up which is isothermal, that is determined at a single temperature but the deformation may be adiabatic. This procedure is shown schematically in Figure 1 where it can be seen that the locus of the $\mathrm{T}_{0}$ points form the isothermal curve.

Isothermal strain hardening curves are essential to make an initial judgement on the form of the model and these curves were determined over a range of temperatures and at two strain rates, $10^{-3} \mathrm{~s}^{-1}$ and $4 \mathrm{~s}^{-1}$. The tests were carried out in tension and large strains were achieved by continuing the tests into the late stages of necking and using a Bridgeman correction. The temperature curves are shown in Figure 2 where it can be seen that the curves are almost parallel. The small divergence is due to the variation in shear modulus with temperature which was discussed by Goldthorpe (4).

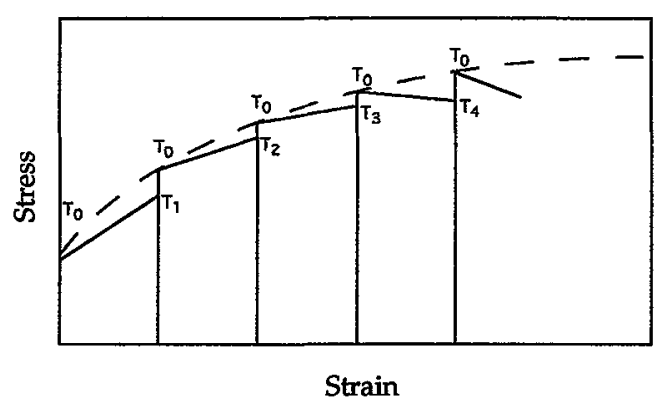

FIG 1. SCHEMATIC INTERRUPTED TESTS

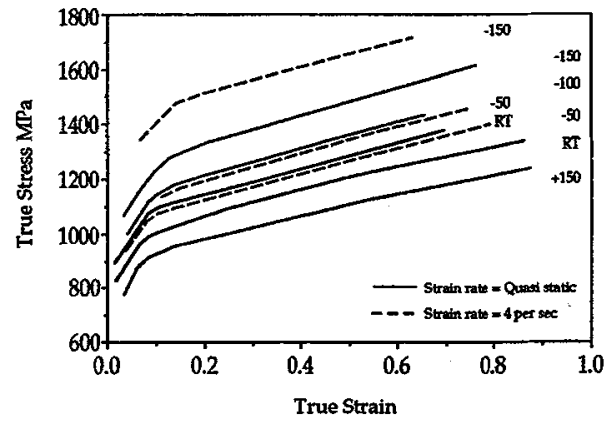

FIG. 2 EFFECT OF TEMPERATURE ON PROPERTIES

The strain rate curves were also found to be parallel and this behaviour suggests that this steel has the same form of model as that for pure iron. This effect can be seen in Figure 2 where the curves at strains of $10^{-3} \mathrm{~s}^{-1}$ and $4 \mathrm{~s}^{-1}$ are compared with the temperature curves. These results are in some ways not surprising in that the two materials have the same crystal structure. The result is nevertheless of considerable interest since we can regard this behaviour as an independence of strain hardening to strain rate and temperature or perhaps from a more fundamental view point that the response of stress to strain rate and temperature in independent of strain and therefore independent of deformed structure. That iron and the present steel with its very much more complex micro structure should behave in the same way is perhaps more surprising when looked at from this viewpoint. 


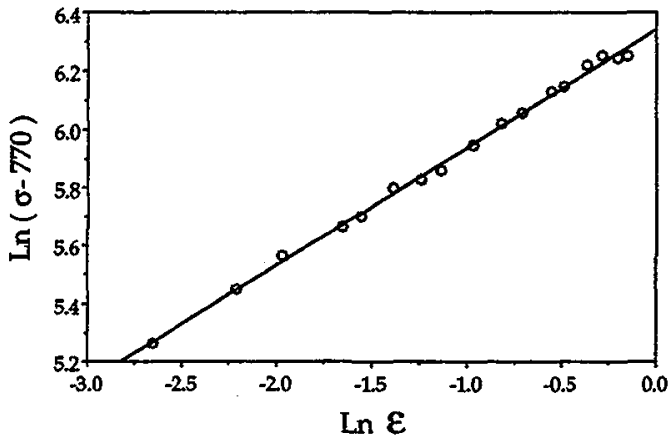

FIG. 3 DETERMINATION OF CONSTANTS

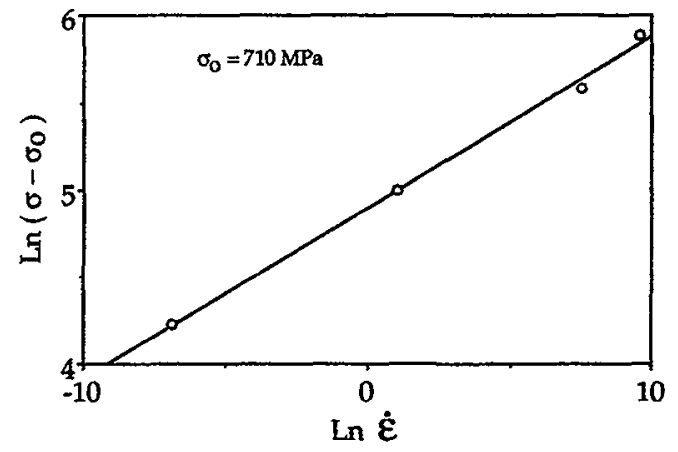

FIG. 4 EFFECT OF STRAIN RATE

Thus the final model is

Table 1

$\begin{array}{cccccc}\mathrm{C}_{1} & \mathrm{C}_{2} & \mathrm{C}_{3} & \mathrm{C}_{4} & \mathrm{C}_{5} & \mathrm{n} \\ 710 & 575 & -0.0048 & 0.00032 & 567 & 0.41\end{array}$

where the stress units are $\mathrm{MPa}$

It is perhaps worth comparing these values with those obtained on pure iron (4). Firstly it was particularly evident that $C_{1}, C_{5}$ and $n$ values in the steel were insensitive to variations in other parts of the experimental data and a high level of confidence can be attached to these values. $C_{2}, C_{3}$ and $C_{4}$ do, however, show a high degree of sensitivity to other parts of the model, particularly the strain hardening and the temperature dependence of the shear modulus. Iron on the other hand showed sufficient independence of the coefficients throughout the model for them all to be determined with a high level of confidence. The principal reason for this appears to be the high value of $710 \mathrm{MPa}$ for $\mathrm{C}_{1}$ in this steel which is interpreted as being the resistance to dislocation movement of major obstacles such as coherent precipitates, compared to $50 \mathrm{MPa}$ for annealed iron and the relatively low value of $\mathrm{C}_{2}$ in the steel. This means that the principal mechanism of thermal softening in this steel is through the temperature dependence of the shear modulus and that the strain rate sensitivity is reduced. Thus the use of equation (5) for the determination of $C_{3}$ and $C_{5}$ requires that two relatively large numbers are subtracted from $\sigma$ on the L.H.S. to leave a relatively small number on the R.H.S. It would appear, therefore, that whereas iron is highly sensitive to the values of $\mathrm{C}_{3}$ and $\mathrm{C}_{4}$ this steel is not and it is this insensitivity that makes the determination of their values more difficult.

Another point of interest is that the coefficients $C_{3}$ and $C_{4}$ would be expected to represent the characteristics of the iron matrix in both iron and steel since they are a measure of the thermal activation processes for dislocation movement. It is, therefore, of some significance that it is only these coefficients which have some similarity in the two models. These are compared below

$$
\mathrm{C}_{3} \quad \mathrm{C} 4
$$

iron $\quad-0.0052 \quad 0.00026$

steel $\quad-0.0048 \quad 0.00032$ 


\section{DETERMINATION OF THE MODEL}

In view of the similarities in behaviour of iron and steel it appeared justified to assume that the model for iron could be used as a basis for the model for steel. This assumption does not involve any pre-judgement since if the assumption is unjustified the linear relationship used to determine coefficients cease to be linear. The model used was as follows:

$$
\sigma=\left(C_{1}+C_{5} \varepsilon^{n}\right) \frac{\mu_{T}}{\mu_{293}}+C_{2} \exp \left[\left(C_{3}+C_{4} \ln \dot{\varepsilon}\right) T\right]
$$

where $\mathrm{C}_{1}$ to $\mathrm{C}_{5}$ and $\mathrm{n}$ are constants and $\sigma, \varepsilon, \dot{\varepsilon}$ and $\mathrm{T}$ are respectively stress, strain, strain rate and temperature in ${ }^{\circ} \mathrm{K} . \mu_{293}$ is the shear modulus at $293^{\circ} \mathrm{K}$ and $\mu_{T}$ is the shear modulus at the current temperature where

$$
\mu_{T}=\mu_{293}(1.13-0.000445 T)
$$

In order to determine the coefficients from the experimental data it is, of course, necessary to assume the form of the model is correct unless or until it is at some stage in the determination proved otherwise.

For compactness we shall write

$$
C_{1}^{1} \equiv C_{1} \frac{\mu_{T}}{\mu_{293}} \quad C_{5}^{1} \equiv C_{5} \frac{\mu_{T}}{\mu_{293}}
$$

Also at constant temperature and strain rate

$$
C_{1}^{1}+C_{2} \exp \left[\left(C_{3}+C_{4} \ln \dot{\varepsilon}\right) T\right]
$$

is constant, say $K(T, \dot{\varepsilon})$ and we can write equation (1) as

$$
\ln [\sigma-K(T, \dot{\varepsilon})]=\ln C_{5}^{1}+n \ln \varepsilon
$$

This gives the relationship between stress and strain in which the L.H.S. is linear with $\ln \varepsilon$ and establishes a unique curve provided strain hardening is independent of strain rate and temperature. Since this has already been established some value of $K(T, \dot{\varepsilon})$ can now be found which gives the best fit straight line. The results of this are shown in Figure 3 which provides the values of $n$ and $C_{5}$ of 0.41 and 567 MPa respectively.

Once the strain hardening coefficients are determined re-arrangement of equation (1) and using (3) gives the form necessary for the determination of the thermal and strain rate coefficients. Thus

$$
\ln \left(\sigma-C_{1}^{1}-C_{5}^{1} \varepsilon^{n}\right)=\ln C_{2}+C_{3} T+C_{4} T \ln \dot{\varepsilon}
$$

If strain rate data measured at constant temperature and strain are, used the value of $C_{1}^{1}$ can be found for the best straight line fit. This is shown in Figure 4 where data at $293^{\circ} \mathrm{K}$ and a strain of 0.1 were used, where the low and intermediate rate tests were from tensile data and the highest rate from Hopkinson bar in compression. This provides the determination of the coefficient $C_{1}^{1}$ and $C_{4}$. In a similar way data at constant strain rate and strain can be used to determine the coefficients $C_{2}$ and $C_{3}$. This is shown in Figure 5 where two sets of data at a strain of 0.1 and strain rates of $10^{-3} \mathrm{~s}^{-1}$ and $4 \mathrm{~s}^{-1}$ were used. 


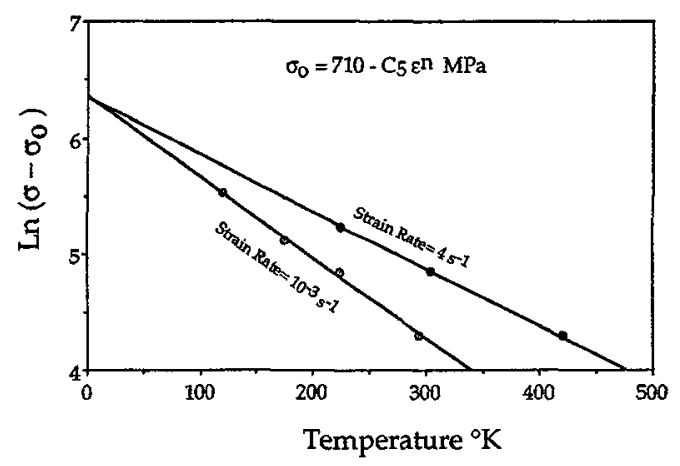

FIG. 5 EFFECT OF THERMAL COEFFICIENTS

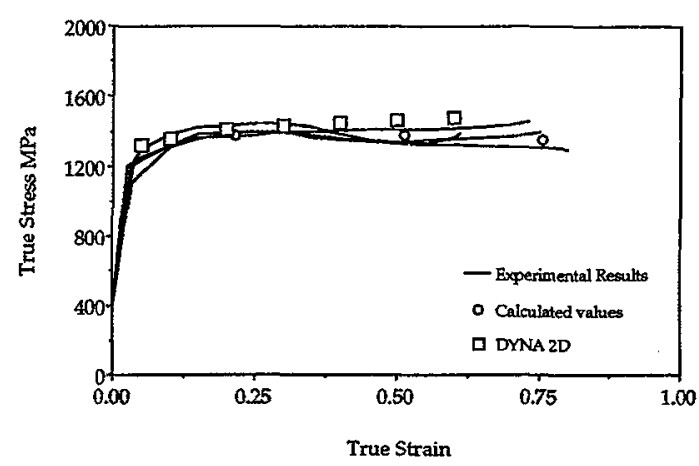

FIG. 6 HOPKINSON BAR RESULTS

\section{VERIFICATION OF THE MODEL}

The simplest method of initial verification of a model is to use a large strain dynamic test. For this a miniature Hopkinson bar was used where strains of about 0.7 were achieved under essentially adiabatic conditions. The experimental curve is shown in Figure 6 and for comparison a calculated curve based on equation (1) and the coefficient values in Table 1. The temperatures were calculated on the assumption of 95\% conversion of mechanical work to heat. It can be seen that the agreement is certainly within the accuracy that is achievable on a large strain Hopkinson bar test. The detailed Hopkinson bar test was also modelled using DYNA 2D and equation (1) and again the agreement between experiment and prediction was good.

Some further modelling simulations were carried out using the DRA Eulerian code GRIM 2D to study the penetration of a target of the steel in the present paper by an EFP made of shocked iron (4). The advantage of this simulation is that models for both materials are known and detailed experimental data were available for comparison. The predicted depth of penetration was $55 \mathrm{~mm}$ compared to an experimental value of $53 \mathrm{~mm}$. The crater diameter at the surface of the target was $50 \mathrm{~mm}$ in the simulation and $46 \mathrm{~mm}$ experimentally. Although the results of one simulation compared to experiment should be treated with some caution it does indicate that for this study predictive capability of the two models used is very good.

\section{DISCUSSION - GENERIC FORMS}

A number of important issues has arisen over the determination of this model for steel. Firstly, it is of a fundamentally different form to any previously published which are mostly based on the Johnson-Cook form. The form of the model was found to follow very closely the behaviour of pure iron in that the strain rate and temperature response of stress are independent of strain. Also the coefficients $C_{3}$ and $C_{4}$ which describe thermal activation are very close to those determined for iron. In spite of this similarity the two materials clearly have very different properties which arise principally through the coefficient $\mathrm{C}_{1}$. This difference is so large that the principal thermal softening mechanism in the steel is through the effect of the shear modulus on $\mathrm{C}_{1}$ and eventually on $\mathrm{C}_{5}$ and $\mathrm{n}$ as strain increases. It would appear, therefore, that as the strength of the iron based alloys (which remain BCC) is increased there is a steady transition from the domination of thermally activated processes to those controlled by the temperature dependence of the shear modulus. Thus strengthening mechanisms such as precipitates which provide obstacles to dislocation movement have their effect through the shear modulus rather than thermal activation. This observation has a number of implications in study of strengthening mechanisms and instabilities such as adiabatic shear where in strong materials instabilities may be controlled by the shear modulus. 
These differences between iron and steel suggest that equation (1) may be used as a generic form for a wide class of BCC iron alloys. If the coefficients $C_{3}$ and $C_{4}$ are assumed to be the same as those for iron the determination of the model would be very much simpler. Thus if we write equation (1) as

$$
\sigma \frac{\mu_{293}}{\mu_{T}}-C_{5} \varepsilon^{n}=C_{1}+C_{2} \exp \left[\left(C_{3}+C_{4} \ln \dot{\varepsilon}\right) T\right] \frac{\mu_{293}}{\mu_{T}}
$$

then if $\mathrm{C}_{3}$ and $\mathrm{C}_{4}$ are known $\mathrm{C}_{1}$ and $\mathrm{C}_{2}$ can be determined from strain hardening data and low strain rate tests over a range of temperatures. This procedure was used on the data available and $C_{1}$ and $C_{2}$ were measured as 715 and $575 \mathrm{MPa}$ compared to the previously determined values of 710 and $575 \mathrm{MPa}$. These changes to $C_{1}, C_{3}$ and $C_{4}$ compared to those in Table 1 have a small effect on predicted stress values which suggests that the model for iron can be used as a generic form for a wide class of iron alloys and this concept of generic forms may simplify the determination of constitutive equations for a large number of other materials.

\section{ACKNOWLEDGEMENTS}

The authors would like to express their thanks to Dr J Field for provision of Hopkinson Bar facilities and to Mr T D Andrews for assistance with other mechanical testing.

\section{REFERENCES}

1 J R Klepaczco

J Mech and Phys of Solids 34 p229 (1986)

C Y Chiem

$2 \quad$ P S Follansbee

International Conference on Impact

Bremen FRG (1987)

$3 \quad$ R W Armstrong

Jnl de Physique 49 C3-529 (1988)

F J Zerilli

4 B D Goldthorpe

Inl de Physique III No 8 C3-829 (1991)

5 I G Cullis

Jnl de Physique III No 8 C3-917 (1991)

P Church 